\title{
A case of drug-coated balloon treatment for three-vessel stenosis with left main bifurcation lesion
}

\author{
Tae-Hyun Kim, Ju-Hyun Chung, Eun-Seok Shin \\ Division of Cardiology, Department of Internal Medicine, Ulsan Hospital, Ulsan, Republic of Korea
}

\begin{abstract}
A 62-year-old man who was a current smoker presented with a 2-month history of effort angina. His treadmill exercise test was positive with significant ST-segment depression at stage 2. Coronary angiography showed a subtotal occlusion of mid right coronary artery (RCA) and $90 \%$ stenosis at the distal left main (LM) bifurcation (Fig. 1A-C). He declined bypass surgery or stent implantation but agreed to treat with drug-coated balloon (DCB) with bailout stenting only in the event of a flow limiting dissection. He was carefully evaluated and provided informed consent.

The RCA lesion was successfully treated with a DCB after balloon angioplasty (Fig. 1D). The lesion of LM to proximal left anterior descending artery (LAD) was dilated with a noncompliant $3.5 \times 15 \mathrm{~mm}$ balloon at $14 \mathrm{~atm}$ and a $3.5 \times 20 \mathrm{~mm}$ DCB was inflated at 8 atm for $60 \mathrm{~s}$. The ostium of the left circumflex (LCX) was not treated. The final angiogram showed normal flow in both LAD and LCX and no significant dissection
\end{abstract}

(Fig. 1E, F). Post-intervention his symptoms resolved, and his follow-up treadmill test was negative.

Six months later, follow-up coronary angiography confirmed adequate patency of the DCB treated segments and reassuringly the LM bifurcation looked better (Fig. 1G-I). He remains symptomfree 12-month post-intervention.

Drug-coated balloon has shown good results in controlling neointimal hyperplasia in the coronary arteries including side branch ostium, although its role in treating LM bifurcation stenosis is still unknown. Even though long-term outcome data are lacking, this case of DCB treatment for distal LM bifurcation stenosis is promising, patency was demonstrated at 6-month, with no evidence of angina at 1-year. These findings suggest that distal LM bifurcation stenosis may be a potential novel indication for DCB, especially when patients are unsuitable for long-term antiplatelet therapy or are unwilling to undergo coronary bypass grafting or stenting.

Conflict of interest: None declared

Address for correspondence: Eun-Seok Shin, MD, PhD, 13, Wolpyeong-ro, 171beon-gil, Nam-gu, Ulsan, 44686, Republic of Korea, tel: +82-52-259-5425, fax: +82-52-259-5117, e-mail: sesim1989@gmail.com 


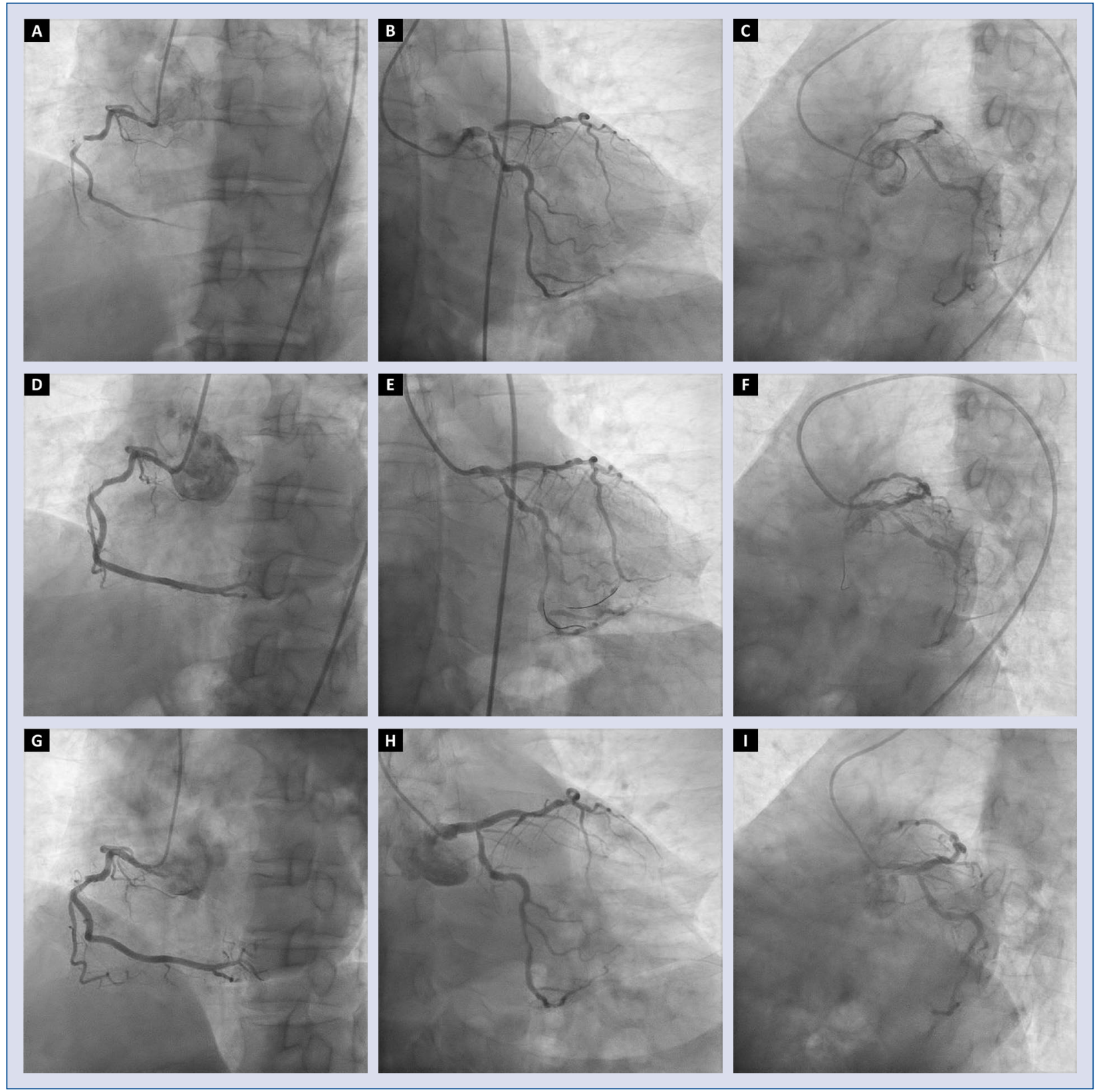

Figure 1. Coronary artery angiography; A-C. Before intervention; D-F. Right after treatment with a drug-coated balloon; G-I. Follow-up angiography at 6-month. 